\title{
NONSTATIONARITY ANALYSIS IN WIND-RAIN-INDUCED VIBRATION OF STAY CABLES
}

\author{
Xu-hui He ${ }^{1}$, Xiang-dong Y $\mathbf{u}^{2}$, Zheng-qing Chen ${ }^{3}$ \\ ${ }^{1,2}$ School of Civil Engineering, Central South University, Changsha, Hunan, China \\ ${ }^{3}$ Wind Engineering Research Center, Hunan University, Changsha, Hunan, China

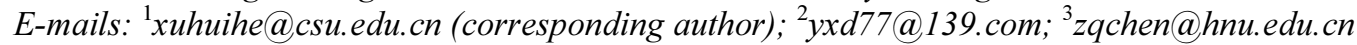

Received 28 Feb. 2011; accepted 09 Apr. 2011

\begin{abstract}
Wind and wind-rain-induced cable vibration are usually nonstationary. Based on the field measured wind data from the Dongting Lake Bridge (DLB) in China, a wavelet-based method was developed to investigate the wind characteristics of wind-rain-induced cable vibration. By using the wavelet analysis, the time-varying mean wind speed is extracted and a nonstationary wind speed model is proposed. The turbulence intensities and probability distributions of measured wind data are calculated using the proposed nonstationary model and compared with those based on empirical mode decomposition (EMD) and design values. The correlation between wind and wind-rain-induced acceleration response are discussed. At the same time, the relativity of acceleration responses of cable and the wind speed are discussed via calculating root mean square (RMS) averaging and acceleration response peak factors.
\end{abstract}

Keywords: wind speed, stay cable, wind-rain induced vibration, nonstationarity, wavelet analysis.

\section{Introduction}

Owing to the structural elegance and relative economy, more and more large-span cable-stayed bridges have been built in recent years throughout the world. However, cables in cable-stayed bridges are prone to vibration induced by weather conditions and the structure (girders and/or towers) to which they are connected due to their large flexibility, relatively small mass and low inherent damping. For example, large amplitude oscillations of stay cables under the simultaneous occurrence of moderate wind and rain conditions have been reported in a number of cable-stayed bridges worldwide (Bosdogianni, Olivari 1996; Chen et al. 2004; Main et al. 2001; Hikami, Shiraishi 1988). This vibration can cause reduced cable and connection life due to fatigue or rapid deterioration of the corrosion protection system and may result in the loss of public confidence in the bridge (Hwang et al. 2009). Intensive studies have thus been carried out to explore the mechanism and explain the complex phenomenon of wind-rain-induced excessive vibration of stay cables. Research on wind-rain-induced cable vibration includes theoretical analyses (Cao et al. 2003; Gu, Lu 2001; Xu, Wang 2003; Yamaguchi 1990), wind tunnel simulation tests (Bosdogianni, Olivari 1996; Gu et al. 2009; Matsumoto et al. 1992) and field observation (Hikami, Shiraishi 1988; Ni et al. 2007; Zuo et al. 2008). Some main features for wind-rain-induced vibration such as occurrence conditions of moderate wind and rain combination, water rivulet formation on upper surface, axial flow in a nearwake of cable, low-frequency vortex shedding along the cable axis, and vortex-induced vibration at high reduced wind speed have been captured (Main et al. 2001). Almost all previous research studies were based on the assumption of considering wind and cable vibration as a stationary random process. In fact, the wind speed and the cable large-amplitude oscillation cannot maintain a stationary level for a long time (Ni et al. 2007).

This paper aims to develop a wavelet-based method to investigate wind and wind-rain-induced response on the basis of field measured wind data from the DLB in China. Combining the field measurements of wind and cable vibration and wavelet multiscale analysis, the timevarying mean wind speed is extracted and a nonstationary wind speed model is proposed based on the typical wind samples. The wind parameters in rain-wind-induced vibration are obtained by using the proposed nonstationary model and compared with those based on EMD and design values. The correlation between cable acceleration response peak factors and mean wind velocity are also discussed. It is concluded that the multiscale-based approach is more appropriate for investigating nonstationary wind and wind-rain-induced vibration of stayed cables.

\section{Description of bridge and field measurements}

The Dongting Lake Bridge (DLB), as shown in Fig. 1, is the first three-tower prestressed concrete cable-stayed bridge located in the influx of Dongting Lake in to the Yangtze River, China. The bridge consists of two main spans of $310 \mathrm{~m}$ each and two side spans of $130 \mathrm{~m}$ each with $25.0 \mathrm{~m}$ clearance height above water level. The deck is $23.4 \mathrm{~m}$ wide with four lanes of traffic. The central tower is $125.7 \mathrm{~m}$ high and side towers are $99.3 \mathrm{~m}$ high each. 
There are a total of 222 cables with size ranging from 28 to $201 \mathrm{~m}$ in length and 99 to $159 \mathrm{~mm}$ in diameter with polyethylene (PE) pipes. Shortly after it opened to traffic in 2000, excessive and unanticipated wind-rain-induced cable vibrations were observed every April, July. The large-amplitude cable vibration cause concerns of the bridge administrative authority and engineers. Unlike the mass dampers used in buliding structures and other type bridges, the MR dampers were finally installed on the cables to mitigate cable vibration after a series of field observation and measurements were conducted (Chen et al. 2004).

The field tests included measurements of wind and rain characteristics, cable vibration and its mitigation by using MR dampers. Two ultrasonic anemometers were installed on the top of the south side tower and deck level near cable A12, respectively. Tower one is situated at an elevation of $102 \mathrm{~m}, 2 \mathrm{~m}$ high above the tower top. Deck level one is situated at an elevation of $26 \mathrm{~m}, 4 \mathrm{~m}$ stretching out from the deck edge with a horizontal cantilever,

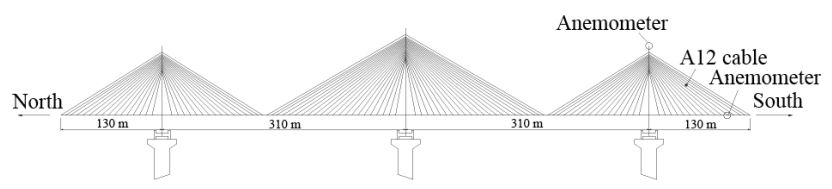

Fig. 1. Elevation of DLB

a)

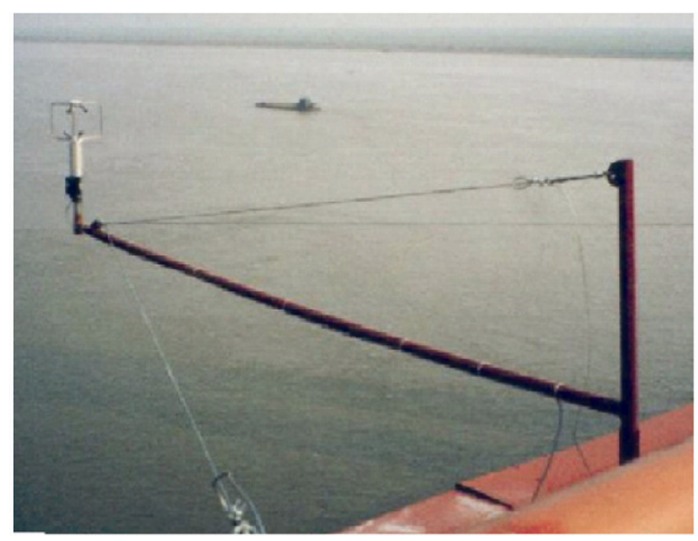

b)

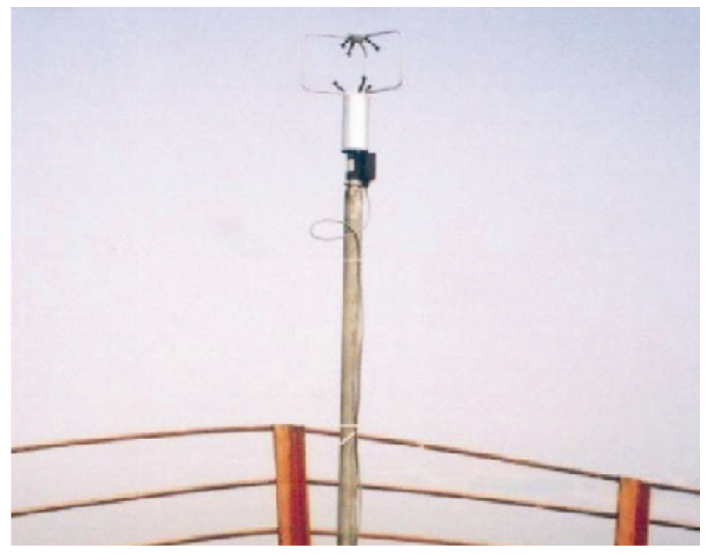

Fig. 2. Ultrasonic anemometers installation for field measurement: (a) anemometer at deck level; (b) anemometer at south side tower top as shown in Fig. 2. Four uniaxial accelerometers were installed on the locations of $\mathrm{L} / 6$ and $\mathrm{L} / 20$ from the lower anchorage for cable A12 in-plane and out-of-plane acceleration measurement. One rain gauge was installed at the deck level near by cable A12. One data acquisition and processing system in the bridge site can record the data while wind-rain-induced vibration occurs. The sample frequency of wind speed and acceleration response are $4 \mathrm{~Hz}$ and $100 \mathrm{~Hz}$, respectively. Continuous field measurements were conducted for 47 days from 24 March to 11 May 2003.

\section{Nonstationary wind speed and time-varying mean wind speed extraction}

Some research studies (Li et al. 2000; Ni et al. 2007) have shown that based on field measurements, wind speed usually has obvious nonstationary characteristics. The characteristics of nonstationary random process represent that the information of time-domain, frequencydomain and so on are related with time and are not ergodic. Thus, it will be unreasonable to assume wind speed or wind pressure as a Gaussian stationary process supposition of in the study of civil engineering. Based on the Gramer theorem, the nonstationary wind speed can be modeled as a deterministic time-varying mean wind speed plus a zero-mean stationary random process for fluctuating wind speed (Xu, Chen 2004):

$$
U(t)=\bar{U}(t)+u(t),
$$

where: $\bar{U}(t)$ is a deterministic time-varying mean wind speed reflecting the temporal trend of wind speed; and $u(t)$ is a fluctuating wind speed of a zero-mean stationary process. The above nonstationary model can be expanded to lateral and vertical wind speed. In fact, the stationary wind speed model Eq. (1) can be looked at as an especial case of the nonstationary model.

The key issue in using nonstationary wind speed model is how to extract the trend in nonstationary signals. The wavelet transform (WT) overcomes the limitations of traditional Fourier transform (FT) and short-time Fourier transform (STFT). It can be thought of as a generalized STFT, with a frequency-dependent window size (Bienkiewicz, Ham 1997). Through dilation of a mother function (wavelet) $\Psi(u)$, adjustment in the window can be accomplished, and localization of frequency resolution is achieved by translation of this function. The resulting wavelet function $\Psi_{\lambda t}(u)$ is thus obtained:

$$
\Psi_{a, b}(t)=\frac{1}{\sqrt{\lambda}} \Psi\left(\frac{t-b}{a}\right),
$$

where $b$ is an instant at which the wavelet function is centered and $a \neq 0$ is a scale parameter controlling the function spread. The signal is then decomposed into a series of basis functions of length consisting of dilated (stretched) and translated (shifted) versions of the mother function, i.e., wavelets of different scales and positions in time or space (Gurley, Kareem 1999). 
Based on the wavelet theory, the wavelet has the character of conservation of energy when the wavelet function is a series of orthogonal basis functions. The wavelet energy of signal scale can be defined as the sum of squares of wavelet coefficients as follows (Zunino et al. 2007):

$$
E_{j}=\left\|r_{j}\right\|^{2}=\sum\left|W_{j, k}\right|^{2}(j=1, \cdots, N) .
$$

For a complex nonstationary signal, the longest period component obtained by WT decomposing in maximum layers, is not always the optimal component reflecting the local information of nonstationary timevarying mean. Therefore, the key issue in using WT to extract the trend in nonstationary signals is how to make sure the most reasonable number of decomposition layers is considered. For a discrete WT, the energy of each layer detail coefficient can be obtained using Eq. (3). The appropriate levels for decomposing the time-varying mean wind speed were quantitatively determined by the sudden change of simple scale wavelet energy.

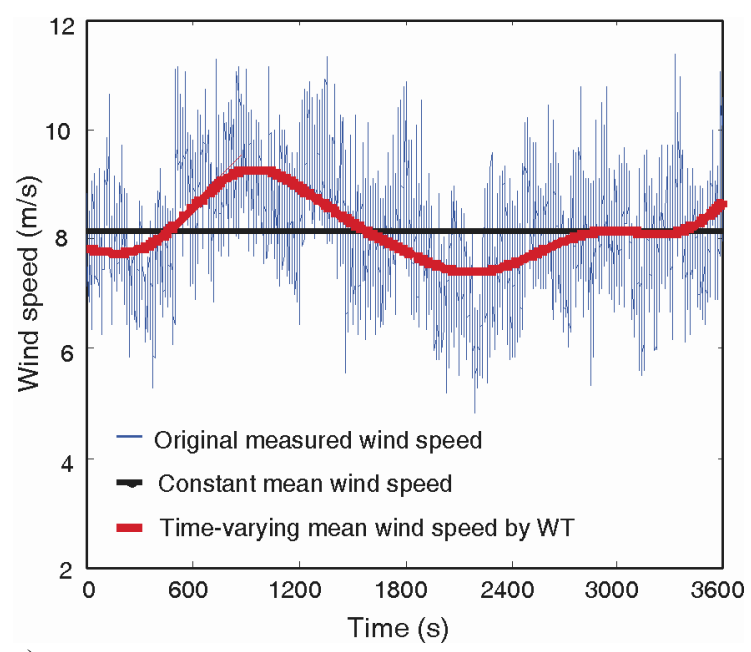

a)

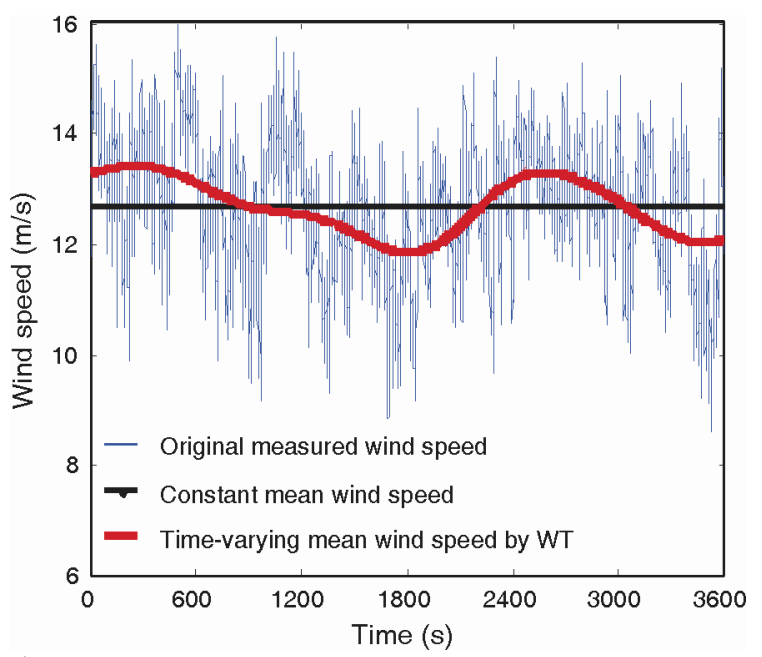

c)

Fig. 3. Typical wind speed and wind direction samples and their time-varying and hourly mean wind speeds: (a) deck level wind speed sample (16:51-17:51, 1 April 2003); (b) deck level wind direction sample (16:51-17:51, 1 April 2003); (c) tower top wind speed sample (16:51-17:51, 1 April 2003); (d) tower top wind direction sample (16:51-17:51, 1 April 2003)

\section{Nonstationary wind characteristics}

\subsection{Wind speed and direction}

The two typical measured wind speed data segments $(1 \mathrm{~h})$ from anemometers installed on the top of the south side tower and bridge deck level during wind-rain-induced vibration duration on 1 April 2003 are considered here. Shown in Fig. $3(\mathrm{a}-\mathrm{b})$ are the $1 \mathrm{~h}$ duration wind speed and wind direction samples from bridge deck level anemometer between 16:51 to 17:51, 1 April 2003, respectively. Shown in Fig. 3 (c-d) are the $1 \mathrm{~h}$ duration wind speed and wind direction samples from the tower top anemometer between 16:51 to 17:51, 1 April 2003. It is seen that the mean wind speed and wind direction in $1 \mathrm{~h}$ is time-varying, and it is not appropriate to adopt the constant mean wind speed assumption. The time-varying mean wind speeds and directions obtained by WT are a continuous function of time with a designated frequency level, which is more natural than the traditional timeaveraged mean wind speed with the certain time interval.

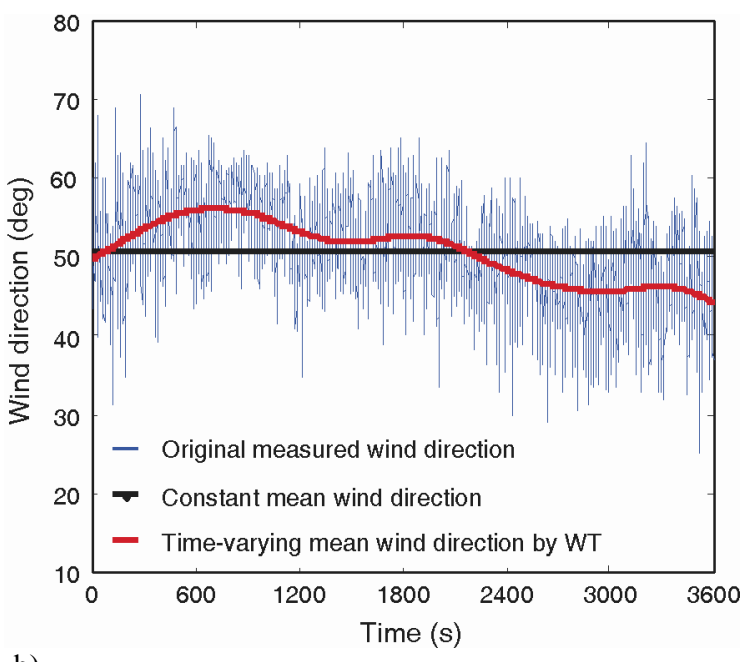

b)

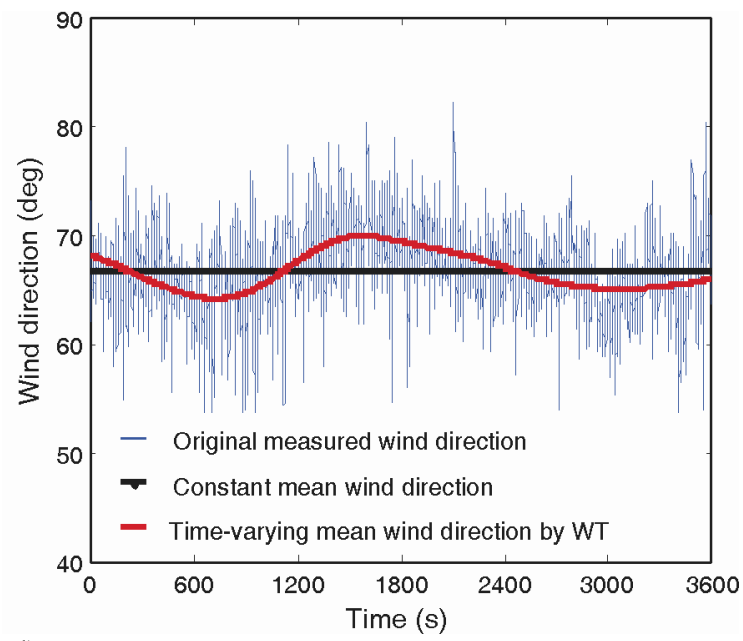

d) 
Using the same method of time-varying mean wind speed extraction, the time-varying mean wind deviation angle and yaw angle are also obtained. The results show that the wind deviation angles of the bridge are nonstationary, the deck level wind deviation angle varied from 30 degree to 60 degree, and the tower top wind deviation angle among 60 degree to 70 degree; but wind yaw angles varied slightly, the deck level wind yaw angle is almost 10 degree, and the tower top windyaw angle is about 20 degree, respectivley.

\subsection{Turbulence intensity}

For stationary wind speed, the ratio of the standard deviation of fluctuating wind to mean wind speed is traditionally defined as the turbulence intensity. For nonstationary wind speed, however, the mean wind speed is time varying, and turbulence intensity is also time dependent over time interval. Table 1 lists the measured turbulence intensities at tower top and deck level during the wind-rain excitation event on April 1-2 2003, where $I_{u}$ indicates the turbulence intensity in the longitudinal direction, $I_{v}$ the turbulence intensity in the lateral direction, and $I_{w}$ the turbulence intensity in the vertical direction. For comparison, the design values based on stationary method (Ni et al. 2007) and corresponding design values are also listed. It is found that the mean values of turbulence intensities computed by nonstationary model are smaller

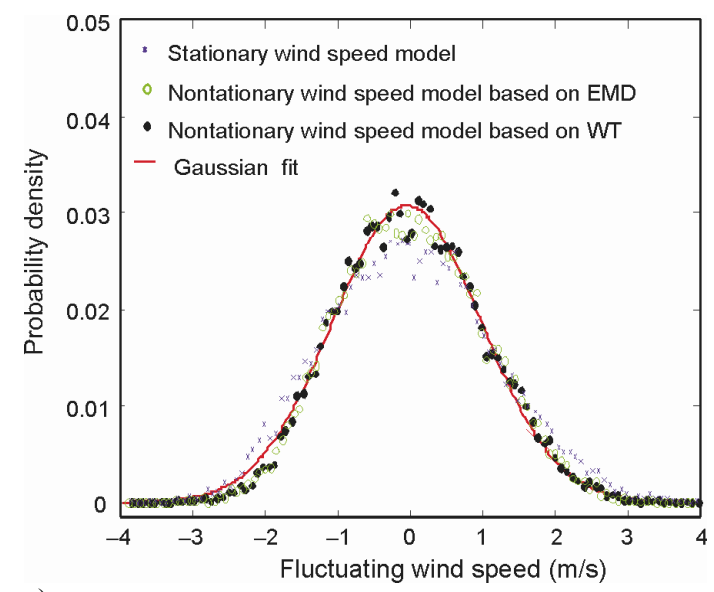

a)

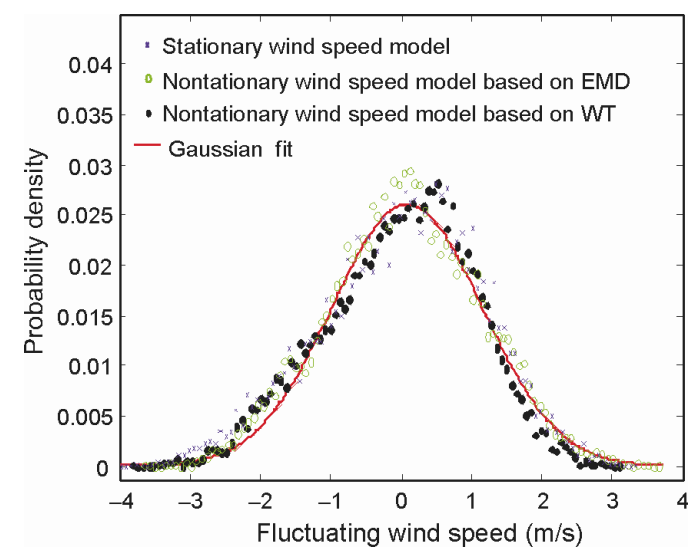

Table 1. Comparison of average values of turbulence intensity

\begin{tabular}{c|c|c|c|c|c}
\hline \multirow{2}{*}{} & & \multicolumn{2}{|c|}{$16: 51-21: 36$} & \multicolumn{2}{c}{$22: 10-02: 48$} \\
\hline \multirow{2}{*}{$\begin{array}{c}\text { Based } \\
\text { on WT }\end{array}$} & $\begin{array}{c}\text { Deck } \\
\text { level }\end{array}$ & $\begin{array}{c}\text { Tower } \\
\text { top }\end{array}$ & $\begin{array}{c}\text { Deck } \\
\text { level }\end{array}$ & $\begin{array}{c}\text { Tower } \\
\text { top }\end{array}$ \\
\cline { 2 - 6 } & $I_{v}$ & 0.084 & 0.0587 & 0.0888 & 0.063 \\
\hline \multirow{2}{*}{$\begin{array}{c}\text { Based } \\
\text { on }\end{array}$} & $I_{w}$ & 0.0815 & 0.0499 & 0.0963 & 0.0677 \\
\cline { 2 - 6 } EMD & $I_{u}$ & 0.1147 & 0.0684 & 0.0802 & 0.0687 \\
\hline \multirow{2}{*}{$\begin{array}{c}\text { Design } \\
\text { value }\end{array}$} & $I_{w}$ & 0.0985 & 0.0631 & 0.1102 & 0.0789 \\
\cline { 2 - 6 } & $I_{u}$ & 0.0871 & 0.0797 & 0.0889 & 0.099 \\
\cline { 2 - 6 } & $I_{v}$ & 0.11 & 0.1 & 0.13 & 0.1 \\
\hline
\end{tabular}

than those obtained by the traditional stationary model and design values. Among the two nonstationary approaches based on WT and EMD, the results by WT are slightly smaller than those by EMD.

\subsection{Probability distribution}

Displayed in Fig. 4 are the probability distributions of the typical fluctuating wind speeds recorded from the deck level and south tower anemometers together with Gaussian density functions, respectively. The probability distributions based on WT are calculated from fluctuating

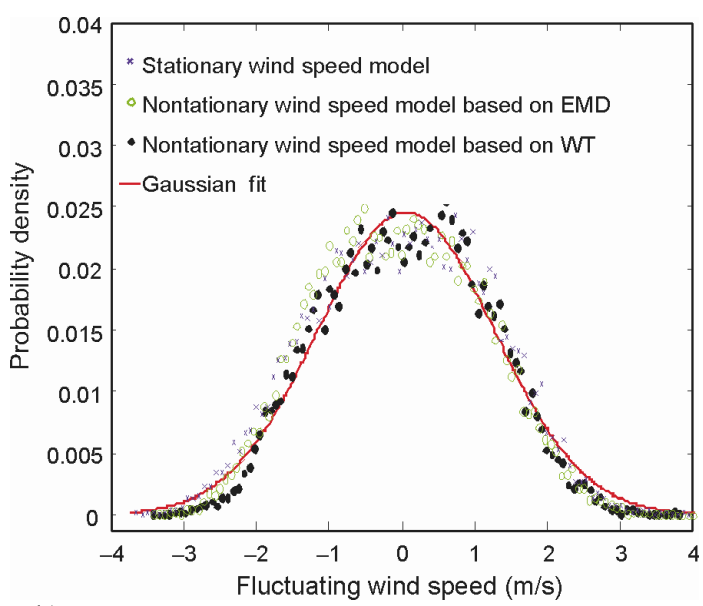

b)

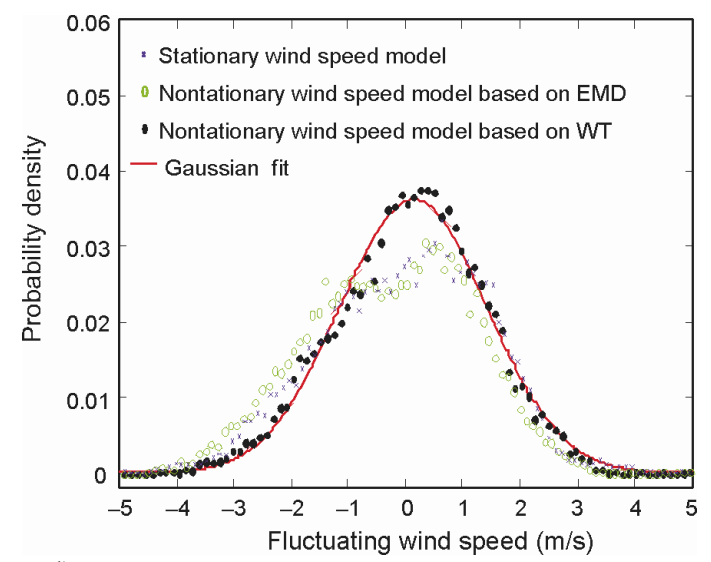

d)

Fig. 4. Comparison of probability densities: (a) deck level wind speed sample (16:51-21:36, 1 April 2003); (b) deck level wind direction sample (22:10-02:48, 1-2 April 2003); (c) tower top wind speed sample (16:51-21:36, 1 April 2003); (d) tower top wind direction sample (22:10-02:48, 1-2 April 2003) 
wind speed obtained by subtracting the time-varying mean wind speed at a frequency level of $1 / 3600 \mathrm{~Hz}$ from original wind sample. The probability distributions based on EMD nonstationary and traditional stationary wind speed models are also computed and compared in Fig. 4. It is seen that the probability densities obtained by the nonstationary model based on WT comply with the Gaussian distribution better than those calculated by nonstationary model based on EMD and traditional stationary model. Especially, as shown in Fig. 4d, the probability distribution of the fluctuating wind speed obtained based WT from tower original wind sample 2 (22:10-02:48, 1-2 April 2003) complies with Gaussian distribution well, but the results obtained based EMD and traditional stationary model deviate from the Gaussian distributions significantly. Thus, it may be concluded that waveletbased stationary model is more reasonable and reliable for characterizing field measured nonstationary wind speeds.

\section{Correlation between wind and wind-rain-induced response}

\subsection{Correlation between wind and response}

Fig. 5 shows the correlations of wind-rain-induced cable A12 in-plane acceleration and wind speed of deck level and tower top. It is seen that the root mean square (RMS) of in-plane accelerations show an increasing tendency with the increase in the RMS of the wind speed of deck level, whereas the relation between the wind speed and out-of-plane acceleration is not very clear. Similar tendencies can be found in correlations of tower top wind fluctuations and RMS of A12 cable out-of-plane accelerations. It is evident from the Fig. 5 that the critical RMS of wind velocity of deck level for wind-rain-induced cable vibration is $7-9.5 \mathrm{~m} / \mathrm{s}$.

\subsection{Relation between response peak factors and mean wind speed}

The response peak factor is defined as the ratio of the maximum value of acceleration to its RMS values and is taken as a parameter to represent the fluctuations in the structural response (Adhikari, Yamaguchi 1997). The relation between the mean wind velocity and response peak factors is shown in Fig. 5. For comparison, the theoretical average peak factors for resonant response based on a narrow band stationary assumption made both on the wind turbulence and structure response are also plotted in Fig. 6. The theoretical values of average peak factors $F$ were calculated by the following equation:

$$
F=\sqrt{2 \ln (n T)}+\gamma / \sqrt{2 \ln (n T)},
$$

where $n$ is the first natural frequency, $T$ is the averaging time and $\gamma$ is the Euler's constant equal to 0.5772 .

As seen in Fig. 6, the peak factors for wind-rain excitation event at deck level during 16:51 to 21:36 of 1 April 2003 show no clearly increasing tendency with increase in the mean wind speed when the averaging time is $10 \mathrm{~min}$ or $1 \mathrm{~min}$, the peak factors are not affected by the increase in mean wind velocity. It is observed that when the averaging time is set to be $10 \mathrm{~min}$, large values of peak factors, as compared to the theoretical values of the peak factors, can occur in the real case, thus highlighting the nonstationary characteristics of the wind turbulence of the cable. Similar results can be found in relation between out-of-plane acceleration peak factors and the mean wind velocity.

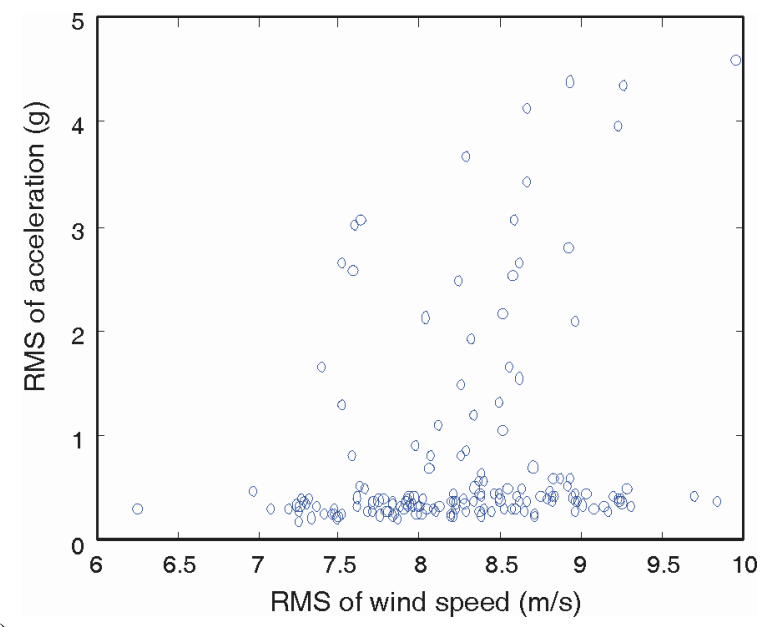

a)

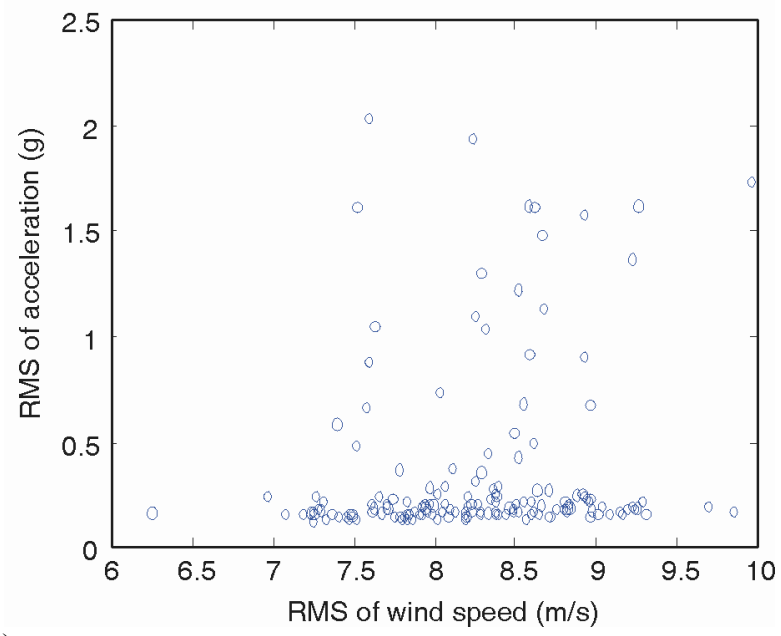

b)

Fig. 5. Correlation between RMS of deck level wind fluctuations and RMS of A12 cable accelerations: (a) In-plane acceleration (16:51-17:51, 1 April 2003); (b) Out-of-plane acceleration (16:51-17:51, 1 April 2003)

\section{Conclusions}

In this paper, the nonstationarity in the wind is discussed from the point of view of developing a wavelet-based method. Based on the analysis of the field measurements of the wind and wind-rain-induced acceleration response of a stayed cable of DLB during wind-rain-induced vibration in April 2003, some conclusions could be drawn as follows:

1. The measured wind speeds as well as the cable response accelerations show remarkable nonstationarity. The nonstationarity in wind may be one of the main factors for the nonstationarity in cable acceleration response. 


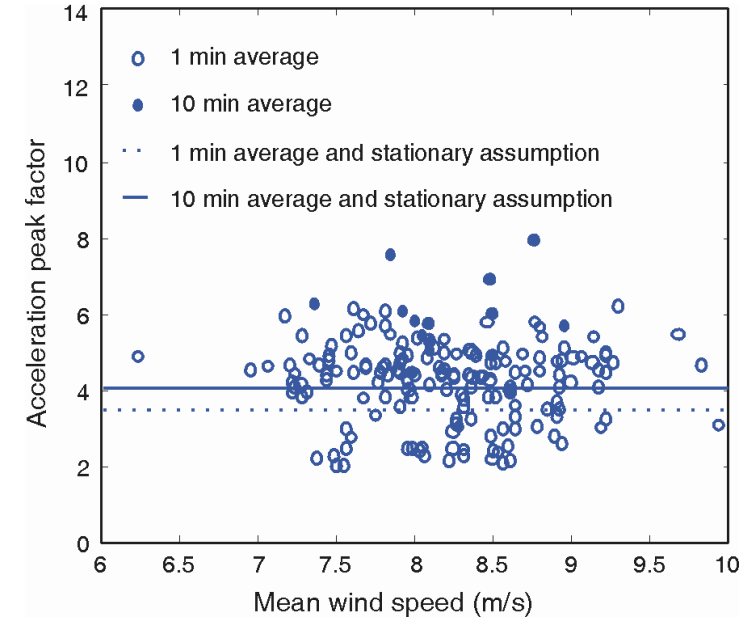

a)

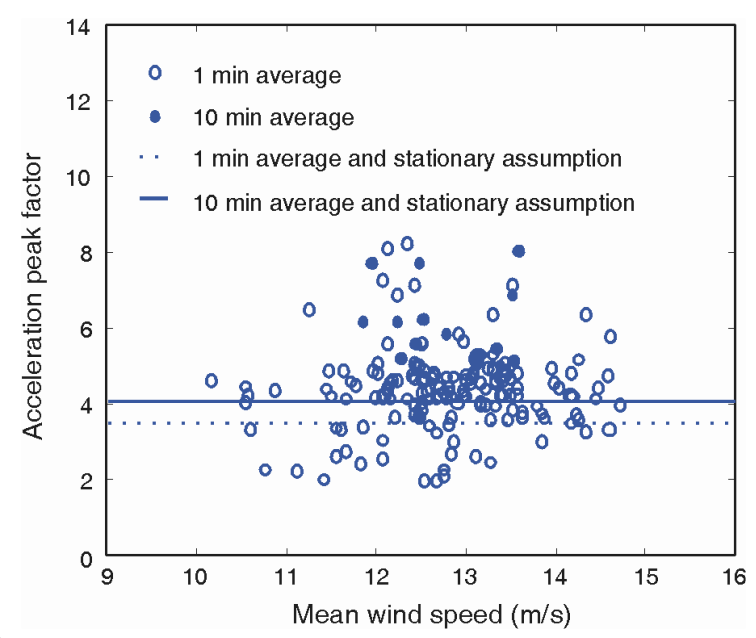

b)

Fig. 6. Relation between in-plane acceleration peak factors and the mean wind speed: (a) deck level wind speed (16:51-21:36, 1 April 2003); (b) tower top wind speed (16:51-21:36, 1 April 2003)

2. The mean values of turbulence intensities calculated by wavelet-based nonstationary model are smaller than those obtained by the traditional stationary model and design values. Therefore, traditional stationary approach may lead to the overestimation of turbulence intensity, and the turbulence intensity estimated by nonstationary model seems to be more reasonable for windrain-induced vibration analyses.

3 . The cable acceleration response peak factors obtained based on nonstatioanry method are generally larger than the theoretical results obtained by stationary assumption. In general, due to wind-rain-induced vibration of stay cables in cable-stayed bridge and wind have obvious nonstionarity, considering wavelet-based analyses is more reasonable for investigating large-amplitude wind-rain-induced vibration of stayed cables.

\section{Acknowledgements}

The work described in this paper was supported by the China National Natural Science Foundations (Grant no. 50808175,51178471$)$ to which the authors gratefully appreciate.

\section{References}

Adhikari, R.; Yamaguchi, H. 1997. A study on the nonstationarity in wind and wind-induced response of tall buildings for adaptive active control, Journal of Wind Engineering and Industrial Aerodynamics 72: 213-224.

http://dx.doi.org/10.1016/S0167-6105(97)00261-4

Bienkiewicz, B.; Ham, H. J. 1997. Wavelet study of approach wind velocity and building pressure, Journal of Wind Engineering and Industrial Aerodynamics 69-71: 671-683. http://dx.doi.org/10.1016/S0167-6105(97)00196-7

Bosdogianni, A.; Olivari, D. 1996. Wind- and rain-induced oscillations of cables of stayed bridges, Journal of Wind Engineering and Industrial Aerodynamics 64(2-3): 171185. http://dx.doi.org/10.1016/S0167-6105(96)00089-X

Cao, D. Q.; Tucker, R. W.; Wang, C. 2003. A stochastic approach to cable dynamics with moving rivulets, Journal of Sound and Vibration 268(2): 291-304. http://dx.doi.org/10.1016/S0022-460X(03)00205-0

Chen, Z. Q.; Wang, X. Y.; Ko, J. M.; Ni, Y. Q.; Spencer, Jr. B. F.; Yang, G.; Hu, J. H. 2004. MR damping system for mitigating wind-rain induced vibration on Dongting Lake cable-stayed bridge, Wind \& Structures 7(5): 293-304.

Gu, M.; Lu, Q. 2001. Theoretical analysis of wind-rain induced vibration of cables of cable-stayed bridges, Journal of Wind Engineering 89: 125-128.

Gu, M.; Du, X. Q.; Li, S. Y. 2009. Experimental and theoretical simulations on wind-rain-induced vibration of 3-D rigid stay cables, Journal of Sound and Vibration 320(1-2): 184-200. http://dx.doi.org/10.1016/j.jsv.2008.07.009

Gurley, K.; Kareem, A. 1999. Applications of wavelet transforms in earthquake, wind and ocean engineering, Engineering Structures 21: 149-167.

Hikami, Y.; Shiraishi, N. 1988. Rain-wind induced vibrations of cables in cable stayed bridges, Journal of Wind Engineering and Industrial Aerodynamics 29(1-3): 409-418. http://dx.doi.org/10.1016/0167-6105(88)90179-1

Hwang, I.; Lee, J. S.; Spencer, Jr. B. F. 2009. Isolation system for vibration control of stay cables, Journal of Engineering Mechanics ASCE 135(1): 62-66. http://dx.doi.org/ 10.1061/(ASCE)0733-9399(2009)135:1(62)

Li, Q. S.; Wong, C. K.; Fang, J. Q.; Jeary, A. P.; Chow, Y. W. 2000. Field measurements of wind and structural responses of a 70-storey tall building under typhoon conditions, The Structural Design of Tall and Special Buildings 9(5): 325-342. http://dx.doi.org/10.1002/1099-1794(200012)9: $5<325::$ AID-TAL169>3.0.CO;2-B

Main, J. A.; Jones, N. P.; Yamaguchi, H. 2001. Characterization of rain-wind induced stay-cable vibrations from full-scale measurements, in Proc. of the $4^{\text {th }}$ International Symposium on Cable Dynamics, May, 2001, Montreal, Canada, 235-242.

Matsumoto, M.; Shirashi, N.; Shirato, H. 1992. Rain-wind induced vibration of cables of cable-stayed bridges, Journal of Wind Engineering and Industrial Aerodynamics 43(1-3): 2011-2022.

http://dx.doi.org/10.1016/0167-6105(92)90628-N

Ni, Y. Q.; Wang, X. Y.; Chen, Z. Q.; Ko, J. M. 2007. Field observations of rain-wind-induced cable vibration in cable-stayed Dongting Lake Bridge, Journal of Wind Engineering and Industrial Aerodynamics 95(5): 303-328. http://dx.doi.org/10.1016/j.jweia.2006.07.001

Xu, Y. L.; Chen, J. 2004. Characterizing nonstationary wind speed using empirical mode decomposition, Journal of Structural Engineering ASCE 130(6): 912-920. http://dx.doi.org/10.1061/(ASCE)0733-9445(2004)130: 6(912) 
Xu, Y. L.; Wang, L. Y. 2003. Analytical study of wind-raininduced cable vibration, Journal of Wind Engineering and Industrial Aerodynamics 91(1-2): 27-40. http://dx.doi.org/10.1016/S0167-6105(02)00333-1

Yamaguchi, H. 1990. Analytical study on growth mechanism of rain vibration of cables, Journal of Wind Engineering and Industrial Aerodynamics 33(1-2): 73-80.

http://dx.doi.org/10.1016/0167-6105(90)90022-5
Zunino, L.; Pérez, D. G.; Garavaglia, M.; Rosso, O. A. 2007. Wavelet entropy of stochastic processes, Physica A: Statistical Mechanics and its Applications 379(2): 503-512.

Zuo, D.; Jones, N. P.; Main, J. A. 2008. Field observation of vortex- and rain-wind-induced stay-cable vibrations in a three-dimensional environment, Journal of Wind Engineering and Industrial Aerodynamics 96(6-7): 11241133. http://dx.doi.org/10.1016/j.jweia.2007.06.046

Xu-hui HE. Is a professor of Civil Engineering in the School of Civil Engineering at Central South University, China. He is a member of IABSE (International Association of Bridge and Structural Engineering), and a member of IABMAS (International Association for Bridge Maintenance and Safety). His interests include bridge wind engineering, bridge structure health monitoring, parameter identification and condition assessment of bridge.

Xiang-dong YU. Is an associate professor of Civil Engineering in the School of Civil Engineering at Central South University, China. His interests include bridge wind engineering, structural design theory and condition assessment of bridge.

Zheng-qing CHEN. Is a professor and director of Wind Engineering Research Center at Hunan University, China. He is a member of ASCE (American Society of Civil Engineers). His interests include bridge wind engineering, nonlinear analysis of large flexible bridge. 